\title{
EIGENVALUES OF HOPF MANIFOLDS
}

\author{
ERIC BEDFORD ${ }^{1}$ AND TATSUO SUWA ${ }^{2}$
}

\begin{abstract}
The eigenvalues of the Laplacians $\Delta$ and $\square$ on the Hopf manifolds are described. Some isospectral results are also given.
\end{abstract}

On a complex manifold $M$ there are the de Rham and Dolbeault complexes, with operators $d$ and $\bar{\partial}$, respectively. If we fix a hermitian metric on the manifold $M$, then $d$ and $\bar{\partial}$ will have formal adjoints $\delta$ and $\delta$ with respect to the hermitian volume element. With these, we define the real Laplacian $\Delta$ $=d \delta+\delta d$ and the complex Laplacian $\square=\bar{\partial} \delta+\delta \bar{\partial}$. We have $\Delta=2$ $=2 \overline{\text { if }} M$ is Kähler.

In this note, we study the eigenvalues of the Laplacians $\Delta$ and $\square$ on the Hopf manifolds $M_{\alpha}, 0<|\alpha|<1$, which are not Kähler. The notation $\mathrm{Sp}(M, A)$ will be used to denote the eigenvalues with multiplicity (spectrum) of the operator $A$ on the manifold $M$. In Theorem 1, the eigenvalues of $\Delta$ and are described. The computation of the eigenfunctions shows that the eigenspaces of $\square$ refine those of $\Delta$. Some isospectral results are also given. For $\alpha$ in a certain domain, $\operatorname{Sp}\left(M_{\alpha}, \Delta\right)$ determines $M_{\alpha}$ up to isometry (Theorem 2). Moreover, it is shown that for "most" values of $\alpha, M_{\alpha}$ may be determined up to isometry from either $\operatorname{Sp}\left(M_{\alpha}, \Delta\right)$ or $\operatorname{Sp}\left(M_{\alpha}, \square\right)$ (Theorem 3).

Let $W$ denote the $n$-dimensional complex vector space $\mathbf{C}^{n}=\{z \mid z$ $\left.=\left(z_{1}, \ldots, z_{n}\right)\right\}$ minus the origin; $W=\mathbf{C}^{n}-\{0\}$, and let $\alpha$ be a complex number with $0<|\alpha|<1$. Consider the analytic automorphism $g_{\alpha}$ of $W$ defined by $g_{\alpha}\left(z_{1}, \ldots, z_{n}\right)=\left(\alpha z_{1}, \ldots, \alpha z_{n}\right)$. The group $G_{\alpha}$ generated by $g_{\alpha}$ is an infinite cyclic group acting on $W$ freely and properly discontinuously. Thus the quotient $M_{\alpha}=W / G_{\alpha}$ is an $n$-dimensional complex manifold, which is called a (homogeneous) Hopf manifold. It is easy to see that $M_{\alpha}$ is diffeomorphic to $S^{1} \times S^{2 n-1}$, where $S^{r}$ denotes the standard $r$-sphere (cf. the proof of Lemma 3). If $n=1, M_{\alpha}$ is the complex torus whose lattice is generated by 1 and $(2 \pi i)^{-1} \log \alpha$, and if $n>1, M_{\alpha}$ is a non-Kähler manifold. The hermitian metric

$$
g=\|z\|^{-2} \sum_{i=1}^{n} d z_{i} d \bar{z}_{i}, \quad\|z\|^{2}=\sum_{i=1}^{n} z_{i} \bar{z}_{i}
$$

Received by the editors June 9, 1975.

AMS (MOS) subject classifications (1970). Primary 53C55, 32C10; Secondary 35P99, 58 G99.

Key words and phrases. Hopf manifold, hermitian metric, Laplacian, eigenvalues, isospectral problem.

${ }^{1}$ Research supported in part by a Sloan Foundation Grant to CIMS and Army Research Office No. DAHC 04-74-G-0159.

2 Research supported in part by NSF Grant GP 38878.

Copyright $\odot 1977$, American Mathematical Society 
on $W$ is $G_{\alpha}$-invariant. Hence, it induces a hermitian metric on $M_{\alpha}$. From now on we think of $M_{\alpha}$ as a hermitian manifold with this metric. It is easy to check that if $n=1, M_{\alpha}$ is a flat torus. If $\alpha$ is real, $M_{\alpha}$ is isometric to $S^{1} \times S^{2 n-1}$ as a riemannian manifold. We denote by $e^{\infty}\left(M_{\alpha}\right)$ the space of complex valued smooth functions on $M_{\alpha}$. Consider the real and complex Laplacians $\Delta$ and $\square$ induced by the hermitian metric on $M_{\alpha}$. Since the operator $\delta$ maps a $(p, q)$ form to a $(p, q-1)$-form, we have

$$
\Delta=\square+\bar{\square} \text { on } e^{\infty}\left(M_{\alpha}\right) .
$$

A straightforward computation [3, p. 97] shows that the complex Laplacian on $\bigodot^{\infty}\left(M_{\alpha}\right)$ is

$$
\square=-\|z\|^{2} \sum_{i=1}^{n} \frac{\partial^{2}}{\partial z_{i} \partial \bar{z}_{i}}+(n-1) \sum_{i=1}^{n} \bar{z}_{i} \frac{\partial}{\partial \bar{z}_{i}} .
$$

Let

$$
\Delta_{0}=-\sum_{i=1}^{n} \frac{\partial^{2}}{\partial z_{i} \partial \bar{z}_{i}}
$$

be the standard Laplacian on $\mathbf{C}^{n}$. Moreover, let $\mathcal{K}_{p, q}$ be the space of harmonic polynomials of type $(p, q)$, i.e., the polynomials $f$ on $\mathbf{C}^{n}$ such that $\Delta_{0} f$ $=0$ and $f(z)=\sum_{|\mu|=p ;|\nu|=q} c_{\mu \nu} z^{\mu} \bar{z}^{\nu}$, where $\mu=\left(\mu_{1}, \ldots, \mu_{n}\right)$ and $\nu$ $=\left(\nu_{1}, \ldots, \nu_{n}\right)$ are $n$-tuples of nonnegative integers, $|\mu|=\sum_{i=1}^{n} \mu_{i},|\nu|$ $=\sum_{i=1}^{n} \nu_{i}$, and $z^{\mu}=z_{1}^{\mu_{1}} \cdots z_{n}^{\mu_{n}}, \bar{z}^{\nu}=\bar{z}_{1}^{\nu_{1}} \cdots \bar{z}_{n}^{\nu_{n}}$.

Lemma 1. For $f \in \mathcal{H}_{p, q}$ and for a complex number $\gamma$,

$$
\square\left(\|z\|^{\gamma} f\right)=\left(-(\gamma / 2)^{2}-(p+q) \gamma / 2+q(n-1)\right)\|z\|^{\gamma} f .
$$

Proof. Since $\Delta_{0} f=0$, we have

$$
\begin{aligned}
\square\left(\|z\|^{\gamma} f\right)= & -\left(\frac{\gamma}{2}\right)^{2}\|z\|^{\gamma} f-\frac{\gamma}{2}\|z\|^{\gamma} \sum_{i=1}^{n} z_{i} \frac{\partial f}{\partial z_{i}} \\
& +\left(n-1-\frac{\gamma}{2}\right)\|z\|^{\gamma} \sum_{i=1}^{n} \bar{z}_{i} \frac{\partial f}{\partial \bar{z}_{i}} .
\end{aligned}
$$

Substituting

$$
\sum_{i=1}^{n} z_{i} \frac{\partial f}{\partial z_{i}}=p f \quad \text { and } \quad \sum_{i=1}^{n} \bar{z}_{i} \frac{\partial f}{\partial \bar{z}_{i}}=q f
$$

in the above equation, we get (3). Q.E.D.

Let $\omega$ be a complex number such that $e^{2 \pi i \omega}=\alpha$. Since $|\alpha|<1$, we have $\operatorname{Im} \omega>0$.

Definition. For nonnegative integers $p$ and $q$, let $\Gamma_{p, q}$ be the set of complex numbers $\gamma$ which can be expressed as $\gamma=-\gamma_{1}+\bar{\gamma}_{2}$ with $\operatorname{Re} \gamma_{1}=p, \operatorname{Re} \gamma_{2}$ $=-q$, and $\operatorname{Re}\left(\left(\gamma_{1}+\gamma_{2}\right) \bar{\omega}\right) \in \mathbf{Z}$.

REMARKS. $1^{\circ} \cdot \gamma_{1}+\gamma_{2}$ is in the lattice dual to the one generated by 1 and $\omega$ (cf. [1, p. 146]). 
$2^{\circ}$. Let $a=\operatorname{Re} \omega$ and $b=\operatorname{Im} \omega$. Then

$$
\Gamma_{p, q}=\{-(p+q)+((a(p-q)-k) / b) i \mid k \in \mathbf{Z}\} .
$$

Lemma 2. For $f \in \mathcal{H}_{p, q}$, the function $\|z\|^{\gamma} f$ is $G_{\alpha}$-invariant if and only if $\gamma \in \Gamma_{p, q}$.

Proof. $\|z\|^{\gamma} f$ is $G_{\alpha}$-invariant if and only if $|\alpha|^{\gamma} \alpha^{p} \bar{\alpha}^{q}=1$, or equivalently,

$$
\left\{\begin{array}{l}
\operatorname{Re} \gamma=-(p+q) \text { and } \\
-\operatorname{Im} \omega \cdot \operatorname{Im} \gamma+(p-q) \operatorname{Re} \omega=k, \quad \text { for some } k \in \mathbf{Z} .
\end{array}\right.
$$

In view of (4), this is equivalent to $\gamma \in \Gamma_{p, q}$. Q.E.D.

LEMMA 3. The vector subspace of $e^{\infty}\left(M_{\alpha}\right)$ generated by $\cup_{p, q \geqslant 0}\left\{\|z\|^{\gamma} f \mid f\right.$ $\left.\in \mathcal{H}_{p, q}, \gamma \in \Gamma_{p, q}\right\}$ is dense in $e^{\infty}\left(M_{\alpha}\right)$.

Proof. Consider the unit $(2 n-1)$-sphere $S^{2 n-1}=\left\{z \in \mathbf{C}^{n} \mid\|z\|=1\right\}$. The map $\varphi: \mathbf{R} \times S^{2 n-1} \rightarrow W$ defined by $\varphi\left(t, z_{1}, \ldots, z_{n}\right)=\left(e^{2 \pi i \omega t} z_{1}, \ldots, e^{2 \pi i \omega t} z_{n}\right)$ is clearly a diffeomorphism. Moreover, $\varphi$ induces a diffeomorphism, which we denote also by $\varphi$, from $S^{1} \times S^{2 n-1}$ onto $M_{\alpha}$, where $S^{1}=\mathbf{R} / \mathbf{Z}$. Take a function $\|z\|^{\gamma} f$ with $f \in \mathcal{H}_{p, q}$ and $\gamma \in \Gamma_{p, q}$. The pullback of $\|z\|^{\gamma} f$ by $\varphi$ is given by

$$
\left(\left(\|z\|^{\gamma} f\right) \circ \varphi\right)(t, z)=\exp (2 \pi i t(i \gamma \operatorname{Im} \omega+p \omega-q \bar{\omega})) f(z)=e^{2 \pi i k t} f(z),
$$

where $k=\operatorname{Re}\left(\left(\gamma_{1}+\gamma_{2}\right) \bar{\omega}\right) \in \mathbf{Z}$. On the other hand, the vector subspace of $e^{\infty}\left(S^{1}\right)$ generated by the functions $\left\{e^{2 \pi i k t}\right\}_{k \in \mathbf{Z}}$ is dense in $e^{\infty}\left(S^{1}\right)$ and the harmonic polynomials are dense in $e^{\infty}\left(S^{2 n-1}\right)$ [1, p. 160]. Q.E.D.

THEOREM 1. (i) The eigenvalues of $\square$ on $\mathrm{C}^{\infty}\left(M_{\alpha}\right)$ are $|\gamma|^{2} / 4+q(n-1), p, q$ $\in \mathbf{Z}^{+}, \gamma \in \Gamma_{p, q}$.

(ii) The eigenvalues of $\Delta$ on $\bigodot^{\infty}\left(M_{\alpha}\right)$ are $|\gamma|^{2} / 2+(p+q)(n-1), p, q \in \mathbf{Z}^{+}$, $\gamma \in \Gamma_{p, q}$, where $\mathbf{Z}^{+}$denotes the nonnegative integers.

Proof. If $\gamma \in \Gamma_{p, q}$, we have $-(\gamma / 2)^{2}-(p+q) \gamma / 2+q(n-1)=|\gamma|^{2} / 4$ $+q(n-1)$. The theorem follows from (2), Lemmas $1-3$, and Lemma A.II.1 in $[1$, p. 143]. Q.E.D

Now let us discuss the isospectral problem. Let $a+b i=\omega=(2 \pi i)^{-1} \log \alpha$ as before. Since $a=(2 \pi)^{-1}$ arg $\alpha$, we may assume that $|a| \leqslant \frac{1}{2}$. Note that if $|a|=\frac{1}{2}$, then $\alpha$ is real and $M_{\alpha}$ is isometric to $S^{1} \times S^{2 n-1}$.

Theorem 2. Suppose (i) $b>1 / 2 \sqrt{2 n+1}$ or (ii) $|a|<b \sqrt{2 n+1}$. Then $\operatorname{Sp}\left(M_{\alpha}, \Delta\right)$ determines $M_{\alpha}$ up to isometry, namely, $\operatorname{Sp}\left(M_{\alpha}, \Delta\right)=\operatorname{Sp}\left(M_{\alpha^{\prime}}, \Delta\right)$ implies $M_{\alpha}$ is isometric to $M_{\alpha^{\prime}}$.

Proof. Let

$$
a^{\prime}+b^{\prime} i=\omega^{\prime}=(2 \pi i)^{-1} \log \alpha^{\prime}, \quad\left|a^{\prime}\right| \leqslant \frac{1}{2} .
$$

If $\operatorname{Sp}\left(M_{\alpha}, \Delta\right)=\operatorname{Sp}\left(M_{\alpha^{\prime}}, \Delta\right)$, then the volumes of $M_{\alpha}$ and $M_{\alpha^{\prime}}$ are the same [1, Corollaire E.IV.2, p. 216], see also [4]. This implies $|\alpha|=\left|\alpha^{\prime}\right|$ or equivalently $b=b^{\prime}$. Consider the eigenvalues 


$$
\lambda\left(p, q, k, a^{(\nu)}\right)=(p+q)^{2} / 2+\left(a^{(\nu)}(p-q)-k\right)^{2} / 2 b^{2}+(p+q)(n-1),
$$

$p, q \in \mathbf{Z}^{+}, k \in \mathbf{Z}$, of $\Delta$ on $e^{\infty}\left(M_{\alpha^{(\nu)}}\right), \nu=0,1, a^{(0)}=a, a^{(1)}=a^{\prime}$. Note that if $p+q \geqslant 2$, then $\lambda\left(p, q, k, a^{(\nu)}\right) \geqslant 2 n$ and that $\lambda\left(p, q, k, a^{(\nu)}\right)=0$ if and only if $p=q=k=0$.

Case (i). $b>1 / 2 \sqrt{2 n+1}$. Since $|a| \leqslant \frac{1}{2}$, we have

$$
\begin{aligned}
\lambda(1,0,0, a) & =a^{2} / 2 b^{2}+n-\frac{1}{2}<2 a^{2}(2 n+1)+n-\frac{1}{2} \leqslant \frac{1}{2}(2 n+1)+n-\frac{1}{2} \\
& =2 n .
\end{aligned}
$$

Similarly, we have $\lambda\left(1,0,0, a^{\prime}\right)<2 n$. Hence, we have

$$
\lambda(1,0,0, a)=\lambda\left(p_{1}, q_{1}, k_{1}, a^{\prime}\right) \text { and } \lambda\left(1,0,0, a^{\prime}\right)=\lambda\left(p_{2}, q_{2}, k_{2}, a\right)
$$

for some $p_{j}, q_{j} \in \mathbf{Z}^{+}$and $k_{j} \in \mathbf{Z}$ with $0 \leqslant p_{j}+q_{j} \leqslant 1, j=1$, 2. If $p_{1}+q_{1}$ $=1$, we have $a^{2}=\left(a^{\prime} \pm k_{1}\right)^{2}$. Since $\left|a^{(\nu)}\right| \leqslant \frac{1}{2}, \nu=0,1$, we get $a^{\prime}= \pm a$. If $p_{2}+q_{2}=1$, we also get $a^{\prime}= \pm a$. If $p_{j}+q_{j}=0, j=1,2$, we have $a^{2}-a^{\prime 2}$ $=k_{1}^{2}-k_{2}^{2}$. Since $\left|a^{2}-a^{\prime 2}\right| \leqslant \frac{1}{2}$, we get $a^{\prime}= \pm a$.

Case (ii). $0<b \leqslant 1 / 2 \sqrt{2 n+1},|a|<b \sqrt{2 n+1}$. We have

$$
\lambda(1,0,0, a)=\frac{a^{2}}{2 b^{2}}+n-\frac{1}{2}<n+\frac{1}{2}+n-\frac{1}{2}=2 n .
$$

On the other hand

$$
\lambda\left(0,0, k, a^{\prime}\right)=\frac{k^{2}}{2 b^{2}} \geqslant 2 k^{2}(2 n+1)>2 n, \text { for }|k| \geqslant 1 .
$$

Hence, we have $\lambda(1,0,0, a)=\lambda\left(p_{1}, q_{1}, k_{1}, a^{\prime}\right)$, with $p_{1}+q_{1}=1$. Thus, we get $a^{\prime}= \pm a$.

If $a^{\prime}=-a$, then $\alpha^{\prime}=\bar{\alpha}$ and the diffeomorphism $W \rightarrow W$ defined by $\left(z_{1}, \ldots, z_{n}\right) \mapsto\left(\bar{z}_{1}, \ldots, \bar{z}_{n}\right)$ induces an isometry between $M_{\alpha}$ and $M_{\alpha^{\prime}}$. Q.E.D.

ThEOREM 3. Suppose $a$ and $b$ are algebraically independent over $\mathbf{Q}$. Then $M_{\alpha}$ may be determined up to isometry from either $\operatorname{Sp}\left(M_{\alpha}, \Delta\right)$ or $\operatorname{Sp}\left(M_{\alpha}, \square\right)$.

Proof. We show that $\operatorname{Sp}\left(M_{\alpha}, \square\right)$ determines $|a|$ and $b$. The same argument with only small modifications will apply to $\operatorname{Sp}\left(M_{\alpha}, \Delta\right)$. Let $\alpha^{\prime}$ be a complex number with $a^{\prime}+i b^{\prime}=(2 \pi i)^{-1} \log \alpha^{\prime},-\frac{1}{2} \leqslant a^{\prime} \leqslant \frac{1}{2}$. We shall assume that $\operatorname{Sp}\left(M_{\alpha}, \square\right)=\operatorname{Sp}\left(M_{\alpha}, \square\right)$ and show that $|a|=\left|a^{\prime}\right|$ and $b=b^{\prime}$. Since the volume of $M_{\alpha}$ determines $b$, and the volume of $M_{\alpha}$ is determined by the asymptotic behavior of the spectrum (see, for instance, Gilkey [4]), it follows that $b=b^{\prime}$. Let

$$
\lambda(p, q, k, a, b)=(p+q)^{2} / 4+(a(p-q)-k)^{2} / 4 b^{2}+(n-1) q .
$$

It is easily seen that the linear span of $\left\{b^{2} \lambda \mid \lambda \in \operatorname{Sp}\left(M_{\alpha}, \square\right)\right\}$ over $Q$ is the linear span over $\mathbf{Q}$ of $\left\{1, a, a^{2}, b^{2}\right\}$. Thus if $\operatorname{Sp}\left(M_{\alpha}, \square\right)=\operatorname{Sp}\left(M_{\alpha^{\prime}}, \square\right)$, then $\left\{1, a, a^{2}, b^{2}\right\}$ and $\left\{1, a^{\prime},\left(a^{\prime}\right)^{2}, b^{2}\right\}$ have the same linear span over $Q$. Thus there exist rationals $r_{j}, r_{j}^{\prime}, 1 \leqslant j \leqslant 4$, such that

$$
a^{\prime}=r_{1}+a r_{2}+a^{2} r_{3}+b^{2} r_{4}, \quad\left(a^{\prime}\right)^{2}=r_{1}{ }^{\prime}+a r_{2}^{\prime}+a^{2} r_{3}^{\prime}+b^{2} r_{4}^{\prime} .
$$


By the algebraic independence of $a$ and $b$, it follows by squaring the left-hand equation that $r_{3}=r_{4}=0$. Thus $a^{\prime}=r_{1}+a r_{2}$.

Now we show that $r_{1}=0, r_{2}= \pm 1$. Observe that if

$$
\operatorname{Sp}\left(M_{\alpha}, \square\right)=\operatorname{Sp}\left(M_{\alpha^{\prime}}, \square\right)
$$

then for integers $(p, q, k)$ there exists $\left(p^{\prime}, q^{\prime}, k^{\prime}\right)$ such that

$$
\lambda(p, q, k, a, b)=\lambda\left(p^{\prime}, q^{\prime}, k^{\prime}, r_{1}+a r_{2}, b\right) .
$$

Conversely, for $\left(p^{\prime}, q^{\prime}, k^{\prime}\right)$ there exists $(p, q, k)$. Multiplying (5) by $b^{2}$ and comparing the coefficients of 1 and $a^{2}$, we obtain

$$
\begin{gathered}
k^{2}=\left(\left(p^{\prime}-q^{\prime}\right) r_{1}-k^{\prime}\right)^{2}, \\
(p-q)^{2}=\left(\left(p^{\prime}-q^{\prime}\right) r_{2}\right)^{2} .
\end{gathered}
$$

Since (7) must always have integer solutions, $r_{2}= \pm 1$. From equation (6) it follows that $r_{1}$ must be an integer, and so $r_{1}=0$ since $-\frac{1}{2} \leqslant a^{\prime} \leqslant \frac{1}{2}$. Thus $|a|$ and $b$ are determined. Q.E.D.

REMARKS. 1. If $n=1, M_{\alpha}$ and $M_{\alpha^{\prime}}$ are biholomorphic, as is well known, if and only if $\left(\begin{array}{l}\omega^{\prime} \\ 1\end{array}\right)=u\left(\begin{array}{l}\omega \\ 1\end{array}\right)$ for some $u \in \operatorname{SL}(2, \mathbf{Z})$, where

$$
\omega=(2 \pi i)^{-1} \log \alpha \text { and } \omega^{\prime}=(2 \pi i)^{-1} \log \alpha^{\prime} .
$$

If $n \geqslant 2, M_{\alpha}$ and $M_{\alpha^{\prime}}$ are biholomorphic, by Hartogs' theorem, if and only if $\alpha^{\prime}=\alpha[2$, Theorem 15.1]

2. It does not seem easy to find the dimension (multiplicity) of each eigenspace. The dimension of $\mathcal{H}_{p, q}$ can be computed as follows. Let $\mathscr{P}_{p, q}$ denote the space of polynomials on $\mathbf{C}^{n}$ of type $(p, q)$ and set $r=\|z\|$. Observe that $\mathcal{K}_{k}$, the harmonic polynomials homogeneous of degree $k$, is subdivided into the spaces $\mathcal{H}_{p, q}$, i.e., $\mathcal{H}_{k}=\bigoplus_{p+q=k} \mathcal{H}_{p, q}$. This may be done since $\Delta_{0}$ maps $\mathscr{P}_{p, q}$ into $\mathscr{P}_{p-1, q-1}$. By repeating the argument in Berger [1, p. 161], we may conclude that for $p \geqslant q$,

$$
\mathcal{P}_{p, q}=\mathcal{K}_{p, q} \oplus r^{2} \mathcal{K}_{p-1, q-1} \oplus \cdots \oplus r^{2 q} \mathcal{H}_{p-q, 0},
$$

and the summands are pairwise orthogonal in $L^{2}\left(S^{2 n-1}\right)$. Thus, $\mathscr{P}_{p, q}=\mathcal{K}_{p, q}$ $\oplus r^{2} \mathscr{P}_{p-1, q-1}$ from which it follows that

$$
\operatorname{dim} \mathcal{H}_{p, q}=\operatorname{dim} \mathscr{P}_{p, q}-\operatorname{dim} \mathscr{P}_{p-1, q-1}
$$

Since

$$
\operatorname{dim} \mathscr{P}_{p, q}=\left(\begin{array}{c}
n-1+p \\
p
\end{array}\right)\left(\begin{array}{c}
n-1+q \\
q
\end{array}\right)
$$

we get

$$
\operatorname{dim} \mathcal{K}_{p, q}=\left(\begin{array}{c}
n+p-1 \\
p
\end{array}\right)\left(\begin{array}{c}
n+q-1 \\
q
\end{array}\right)-\left(\begin{array}{c}
n+p-2 \\
p-1
\end{array}\right)\left(\begin{array}{c}
n+q-2 \\
q-1
\end{array}\right) .
$$




\section{REFERENCES}

1. M. Berger, P. Gauduchon and $\mathrm{E}$. Mazet, Le spectre d'une variété riemannienne, Lecture Notes in Math., vol. 194, Springer-Verlag, Berlin and New York, 1971. MR 43 \#8025.

2. K. Kodaira and D. C. Spencer, On deformations of complex analytic structures. II, Ann. of Math. (2) 67 (1958), 403-466. MR 22 \#3009.

3. J. Morrow and K. Kodaira, Complex manifolds, Holt, Rinehart and Winston, New York, 1971. MR 46 \#2080.

4. P. Gilkey, The spectral geometry of real and complex manifolds, Proc. Sympos. Pure Math., vol. 27, Amer. Math. Soc., Providence, R. I., 1975, pp. 265-280.

Courant Institute of Mathematical Sciences, New York University, New York, New YORK 10012 